EXPERIMENTAL STUDY

\title{
Dual control of cytochrome-c oxidase activity by female sex steroids
}

\author{
Maria Abelenda and Marisa Puerta \\ Department of Animal Biology II (Physiology), Faculty of Biological Sciences, Complutense University, 28040 Madrid, Spain \\ (Correspondence should be addressed to M Abelenda; Email: abelenda@eucmax.sim.ucm.es)
}

\begin{abstract}
Female sex steroids modify cytochrome-c oxidase (COX) activity in brown adipose tissue. To check the possibility of extending this modulating effect upon oxidative capacity to other tissues, COX activity was measured in different tissues from cold-acclimated female rats that were (1) intact in proestrus and diestrus I, (2) ovariectomized or (3) ovariectomized and treated with oestradiol and/or progesterone. In intact rats, COX activity varied within the oestrous cycle in brown adipose tissue and soleus muscle. Ovariectomy induced an increase in COX activity in most of the tissues studied, an increase reversed only after 10 days of treatment with oestradiol and/or progesterone. These results indicate both a short-term (oestrous cycle) and a long-term (ovariectomy) control of COX activity by female sex steroids, probably mediated by allosteric modulation and control of the enzyme synthesis respectively. In thermogenic tissues, that is brown adipose tissue and skeletal muscles, the short-term control is interpreted as a cooperation between tissues to fulfil the requirements of temperature maintenance.
\end{abstract}

European Journal of Endocrinology 141 630-636

\section{Introduction}

Cold acclimation in rats induces an increase in oxygen consumption and thus in oxidative activity which allows an enhanced heat production. Most of it is due to the activation of non-shivering thermogenesis in brown adipose tissue (BAT); in this tissue, the uncoupling of the oxidative phosphorylation by the uncoupling protein UCP1 sustains the increased oxidative activity (1). However, other tissues, such as muscle, have also been implicated in non-shivering thermogenesis $(2,3)$. The recent finding of new members of the UCP family, UCP2 and UCP 3 , present in tissues other than BAT, and especially in skeletal muscles $(4,5)$, has led to new interest in this issue.

Non-shivering thermogenesis in BAT is modulated by different hormonal systems, female sex steroid hormones being one of them. Oestradiol treatment in cold-acclimated female rats induces a decrease in both GDP binding and oxidative capacity, an effect not shown when the animals are maintained in the thermoneutral zone (6). On the other hand, progesterone treatment does not modify cold-induced thermogenesis as oestradiol does, but it impairs diet-induced thermogenesis in BAT (7). Recently, it has been shown that oxidative capacity in BAT changes not only with long-term hormonal treatments, but also with the endogenous rhythm of sex steroids during the oestrous cycle. Thus, in cold-acclimated female rats, cytochrome-c oxidase
(COX) activity reaches a lowest level in proestrus and a highest in diestrus I (8), just when oestradiol and progesterone are at their maximum and minimum plasma levels respectively (9). This fluctuation in COX activity is not mirrored by a fluctuation in thermogenic activity, since GDP binding does not change significantly throughout the oestrous cycle (8).

Taken together, these results point out that female sex steroids inhibit oxidative capacity in BAT quite soon, even before the inhibition of thermogenic activity takes place. This raises the possibility that sex steroid hormones also regulate COX activity in other tissues, especially in those likely involved in thermogenesis, such as skeletal muscles. Since this COX modulation in BAT has been shown only when oxidative activity is at its highest, it seems that cold-acclimated rats could be an excellent physiological model to study the effects of sex steroids upon oxidative capacity in different tissues. Accordingly, we have studied COX activity in different tissues from cold-acclimated female rats that were intact, ovariectomized (OVX), or OVX and treated with oestradiol and/or progesterone to check the hypothesis of a general control of oxidative capacity by sex steroids.

\section{Materials and methods}

Female Wistar rats weighing 200-220 g at the beginning of the experiment were maintained in a cold-room 
at $6 \pm 1{ }^{\circ} \mathrm{C}$, housed in single cages with water, allowed to feed (A04, Panlab, Barcelona, Spain) ad libitum and kept with a ratio of $12 \mathrm{~h}$ light: $12 \mathrm{~h}$ darkness (lights on at $0800 \mathrm{~h}$, lights off at $2000 \mathrm{~h}$ ). Animals were cared for and used in accordance with the principles of The Council of European Communities (86/609 EEC).

Intact animals were cold-acclimated for 14 days. From that day onward, vaginal smears were taken daily, animals being sacrificed either in proestrus (group P) or diestrus I (group DI). At the time of sacrifice they had spent 24-29 days in the cold.

To study the effects of ovariectomy and hormonal replacements, rats were bilaterally ovariectomized under light ether anaesthesia after 14 days in the cold. Ten days later, they received one of the following subcutaneous implants under light ether anaesthesia. Group OVX: an empty Silastic capsule; rats were sacrificed 2 days later. Groups OVX + E: a 17- $\beta$ oestradiolfilled Silastic capsule; animals were sacrificed either 2 days (group OVX + E2) or 10 days (group OVX + E10) later. Groups OVX + P: two progesterone-filled Silastic capsules; animals were sacrificed either 2 days (group OVX + P2) or 10 days (group OVX + P10) later. Group OVX + E2P: a $17 \beta$-oestradiol-filled Silastic capsule plus a single subcutaneous injection of progesterone $(200 \mu \mathrm{g} /$ $0.1 \mathrm{ml}$ sesame oil) 2 days later; sacrifice took place $6 \mathrm{~h}$ after progesterone injection. Finally, group OVX + E10P: a $17 \beta$-oestradiol-filled capsule plus two progesteronefilled Silastic capsules 6 days later; animals were sacrificed 4 days later. The length of Silastic capsules was either $0.5 \mathrm{~cm}$ for $17 \beta$-oestradiol-filled ones or $4 \mathrm{~cm}$ for progesterone-filled capsules. Rats remained in the cold during hormonal treatments until sacrifice.

In all groups, vaginal smears were taken daily from ovariectomy to the end of the experiment to check the effectiveness of ovariectomy (diestrus-like smears), oestradiol treatment (oestrus-like smears) and progesterone treatment (diestrus-like smears). Animals were weighed both at the beginning and at the end of the experiment, and on the days of surgical manipulations.

Animals were sacrificed by decapitation, and tissues were quickly removed and stored at $-85^{\circ} \mathrm{C}$ until measurements. Samples from the following tissues were taken: interscapular BAT; heart (ventricle); skeletal muscles: diaphragm, soleus, trapezius, pectoralis, gluteus, vastus lateralis, obliquus abdominis and rectus abdominis; smooth muscles: stomach (body region), duodenum, colon, rectum and uterus; liver and kidney.

A portion of each tissue was homogenized in cold phosphate buffer $0.01 \mathrm{~mol} / \mathrm{l}, \mathrm{pH} 7.0$, centrifuged twice at 3500 r.p.m., $4{ }^{\circ} \mathrm{C}$, and COX activity was immediately measured in the combined supernatants by the technique of Yonetani and Ray (10). Protein concentration was measured by the method of Lowry et al. (11). In the case of OVX-treated groups, only those tissues in which ovariectomy modified COX activity with respect to intact groups were analysed, except for uterus.
Two different statistical analysis were carried out. Differences among groups P, DI and OVX were evaluated by one-way ANOVA. The effects of hormonal replacements in OVX animals were evaluated by two-way ANOVA, the two factors being treatment and time. Posthoc comparisons were made using the StudentNewman-Keuls method. When only two groups were compared (body weight gain), a Student's t-test was used. In all cases, $P<0.05$ was considered as statistically significant.

\section{Results}

Body weight gain did not differ in intact female rats in proestrus and diestrus I after $24-29$ days at $6{ }^{\circ} \mathrm{C}$ $(+19 \pm 5 \mathrm{~g}$ versus $+23 \pm 4 \mathrm{~g}, n=7)$. In contrast, 10 days after ovariectomy, there was a statistically significant increase of body weight gain in all the OVX rats with respect to intact rats during the same period $(+25 \pm 1 \mathrm{~g}, n=51$ versus $+6 \pm 2 \mathrm{~g}, n=14)$. Two-way ANOVA indicated an effect of time and treatment in OVX hormonally treated groups; progesterone treatment increased body weight gain $(+4 \pm 1 \mathrm{~g}$ and $+23 \pm 5 \mathrm{~g}$ in groups OVX $+\mathrm{P} 2$ and OVX $+\mathrm{P} 10$ respectively), whereas oestradiol treatment lowered it $(-8 \pm 2 \mathrm{~g}$ and $-2 \pm 2 \mathrm{~g}$ in groups OVX $+\mathrm{E} 2$ and OVX + E10 respectively). Treatment with both oestradiol and progesterone had the same effect as oestradiol treatment $(-11 \pm 1 \mathrm{~g}$ and $-1 \pm 2 \mathrm{~g}$ in OVX $+\mathrm{E} 2 \mathrm{P}$ and OVX + E10P respectively).

COX activity in BAT is shown in Fig. 1. As expected, in intact rats COX activity was significantly greater in diestrus I than in proestrus. Ovariectomy produced a dramatic increase in such activity, reaching values twice those found in proestrus. In OVX groups, two-way ANOVA revealed main effects of both treatment and time, in such a way that treatments with oestradiol and/or progesterone reversed the increase in COX activity induced by ovariectomy after 10 days, but not after 2 days.

Figure 2 shows COX activity in heart and different skeletal muscles. Decreasing values of COX activity in intact groups was chosen as a criteria from the top to bottom panels. A statistically significant difference between groups P and DI was only found in soleus muscle, with a greater activity in the $\mathrm{P}$ group, whereas the oestrous cycle did not modify COX activity in the remaining sampled muscles. Ovariectomy induced an increase in COX activity with respect to intact groups in heart, diaphragm, soleus, trapezius, pectoralis and gluteus muscles. Nonetheless, it had no effect in vastus lateralis, obliquus abdominis and rectus abdominis muscles. Two-way ANOVA revealed effects of the three treatments tested as well as an effect of time in heart, diaphragm, trapezius, pectoralis and gluteus. On the contrary, in the soleus muscle only 10 days of oestradiol plus progesterone treatment reduced COX activity. 


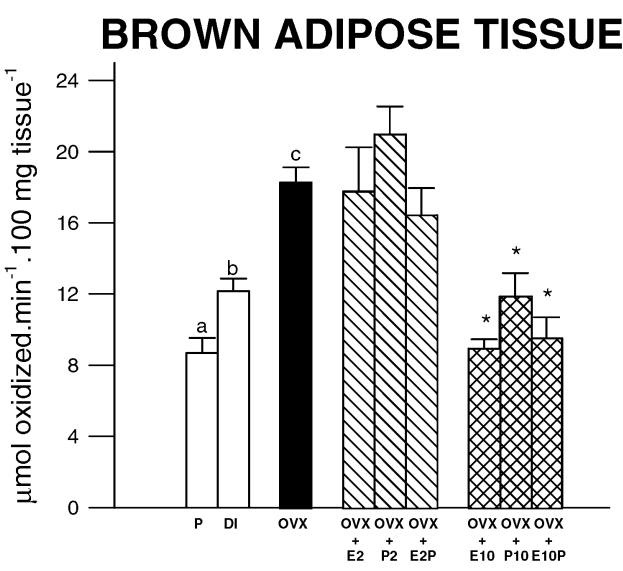

Figure 1 COX activity in brown adipose tissue from female cold-acclimated rats: intact in proestrus $(P)$ and diestrus I (DI); ovariectomized (OVX); ovariectomized and treated for 2 days or for 10 days with either oestradiol $(\mathrm{OVX}+\mathrm{E} 2$ and OVX $+\mathrm{E} 10$ respectively), progesterone (OVX $+\mathrm{P} 2$ and OVX $+\mathrm{P} 10$ respectively) or oestradiol and progesterone (OVX + E2P and OVX + E10P respectively). In intact rats, COX activity was cyclic with higher values in DI than in P. Ovariectomy induced an increase that was reversed by 10 days of hormonal treatments. Bars are means \pm S.E. of seven animals per group. Among groups $P$ DI and OVX, bars with different superscript letters are statistically different $(P<0.05$, one-way ANOVA); among OVX groups, *indicates a statistical difference with respect to OVX group $(P<0.05$, two-way ANOVA).

Figure 3 shows COX activity in several smooth muscles (the panels have again been ordered according to total values of COX activity in intact animals). It also shows that the oestrous cycle does not change such activity in the muscles studied. Ovariectomy increased significantly COX activity in stomach and duodenum, but not in the other smooth muscles. In both stomach and duodenum, either oestradiol, progesterone or oestradiol plus progesterone treatments induced a clear decrease in COX activity after 10 days. An unexpected result was obtained in the uterus. Thus, while COX activity was not altered 10 days after OVX, it was increased 2 days later, despite short-term hormonal treatments. However, 10 days of whichever hormonal treatment led COX activity to values similar to those in intact animals.

Figure 4 shows COX activity in the kidney and liver. As in smooth muscles, the oestrous cycle did not change COX activity, whereas ovariectomy increased it only in kidney. Contrary to results obtained in most of the tissues analysed, either oestradiol, progesterone or both were ineffective to decrease COX values to those of intact rats.

Table 1 shows the protein content of all the studied tissues. It only changed during the oestrous cycle in BAT and liver, gluteus muscle being the only tissue affected by ovariectomy. None of the hormonal treatments had an effect on protein content of analysed tissues.

\section{Discussion}

This study shows that the total absence of female sex steroids in OVX animals induces a generalized stimulation of COX activity. In fact, ovariectomy increased COX activity in 10 of the 17 tissues analysed in this study, including BAT (Fig. 1), heart, skeletal muscles (Fig. 2), smooth muscles (Fig. 3) and kidney (Fig. 4). If we take into account that the tissues where the effect of ovariectomy was statistically significant were those with higher COX activity, it seems reasonable to think that the effects of ovariectomy in tissues with lower oxidative capacity may have been undetected either because of their low basal oxidative capacity and/ or because of the sensitivity of the method used. Therefore, the effect of ovariectomy on COX activity seems to be general, suggesting that in the adult female either oestradiol or progesterone or both control the oxidative capacity in a wide range of tissues. To check such a conclusion, we treated OVX animals with either progesterone, oestradiol or both hormones. Two-day treatments were ineffective in most of the tissues tested (Figs 1-4). However, 10 days of treatment reversed the increases in COX activity brought about by ovariectomy, bringing COX activity values to the levels seen in intact rats (Figs 1-4) (the only exception was kidney where even after 10 days of treatment, COX activity remained high). Both hormones, oestradiol and progesterone, either alone or in combination, produced a similar decrease (in soleus muscle both hormones were needed for decreasing COX activity to preovariectomy values). Accordingly, these results show that female sex steroids control the oxidative capacity of tissues and therefore are a main drive in the whole body oxidative metabolism, a fact that we suggested previously (12) but that only now has been unequivocally demonstrated. Nonetheless, since plasma levels of these hormones change on a daily basis during the oestrous cycle, we also looked for changes in COX activity during the oestrous cycle. In agreement with the results of a recent study (8), COX activity in BAT from cold-acclimated female rats varied throughout the oestrous cycle, with higher values in diestrus I than in proestrus (Fig. 1). Since diestrus I is the oestrous cycle phase with the lowest plasma oestradiol and progesterone levels, whereas in proestrus both oestradiol and progesterone are at their maximal levels (9), those results agree with those found in OVX rats where the lack of sex hormones enhanced BAT activity. However, from all the other tissues studied only the soleus muscle showed differences in COX activity throughout the oestrous cycle (Figs 2-4). Surprisingly, in this muscle the COX activity cycle was opposite to that found in BAT, i.e. the activity was at its maximum in proestrus and at its minimum in diestrus I.

To analyse both sets of results, i.e. the ovariectomyhormonal treatments and the oestrous cycle, the structure of COX should be considered. This enzyme 

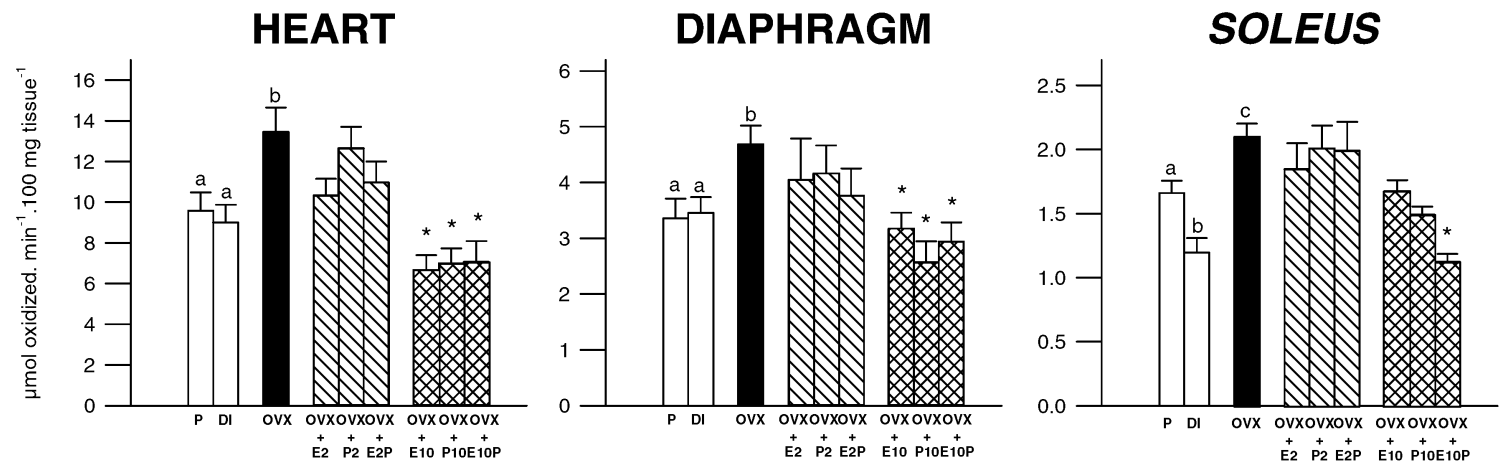

TRAPEZIUS
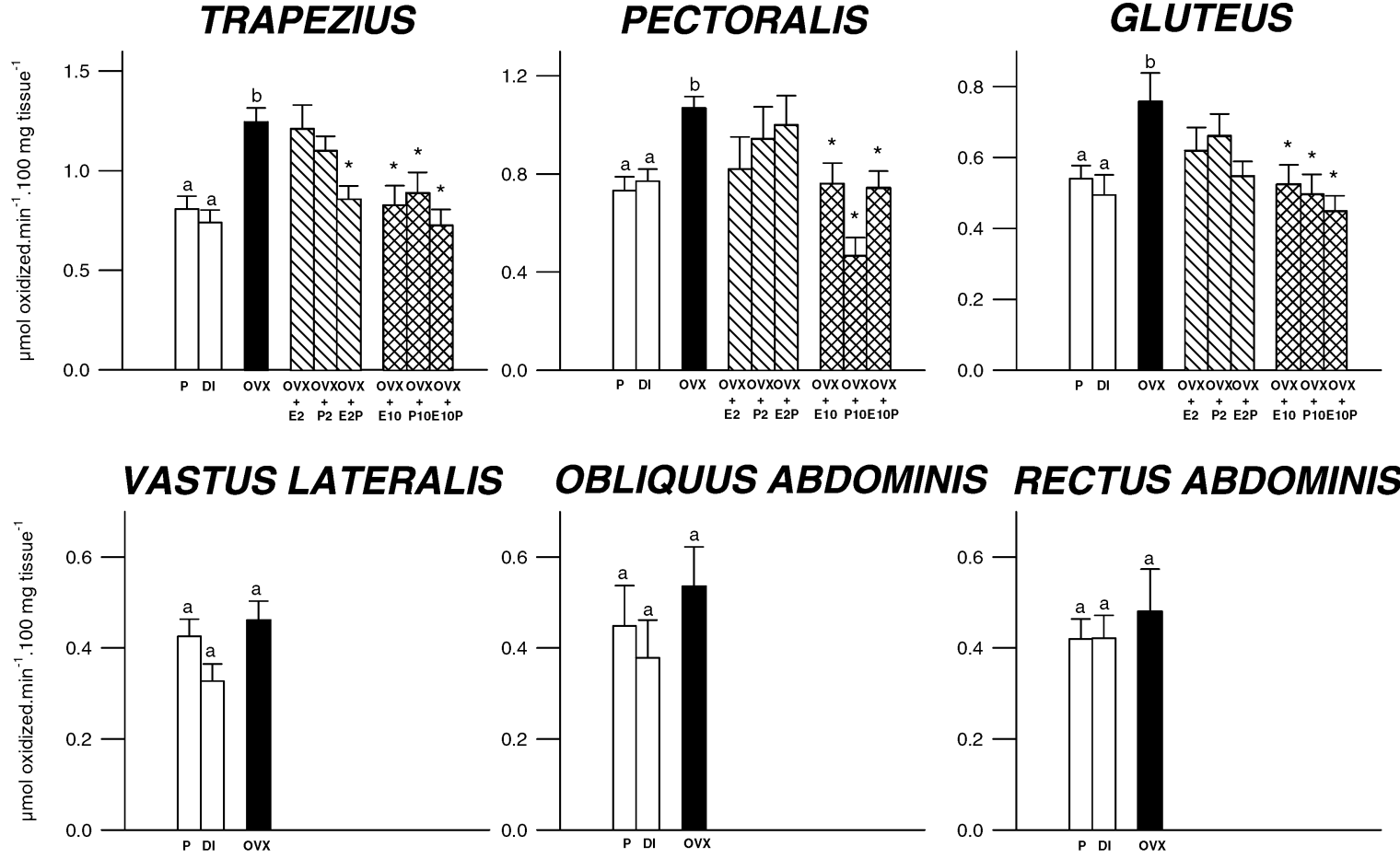

Figure 2 COX activity in heart and skeletal muscles from female cold-acclimated rats: intact in proestrus (P) and diestrus I (DI); ovariectomized $(\mathrm{OVX})$; ovariectomized and treated for 2 days or for 10 days with oestradiol $(\mathrm{OVX}+\mathrm{E} 2$ and $\mathrm{OVX}+\mathrm{E} 10$ respectively), progesterone (OVX $+\mathrm{P} 2$ and $\mathrm{OVX}+\mathrm{P} 10$ respectively) or oestradiol and progesterone (OVX $+\mathrm{E} 2 \mathrm{P}$ and $\mathrm{OVX}+\mathrm{E} 10 \mathrm{P}$ respectively). In intact rats, COX activity was cyclic in soleus with higher values in $\mathrm{P}$ than in DI. Such a cycle was not recorded in the other muscles studied, either heart or skeletal muscles. Ovariectomy induced an increase in most of the muscles studied. This increase was generally reversed by 10 days of hormonal treatments. Bars are means \pm S.E. of six to seven animals per group. Among groups $\mathrm{P}, \mathrm{DI}$ and OVX, bars with different superscript letters are statistically different $\left(P<0.05\right.$, one-way ANOVA); among OVX groups, ${ }^{*}$ indicates a statistical difference with respect to OVX group $(P<0.05$, two-way ANOVA).

contains as many as 13 subunits in mammals. Subunits I, II and III are coded by mitochondrial DNA and have the catalytic core. Subunits IV-VIII are coded by nuclear DNA and they regulate the tissue rate of respiration either modulating catalysis or stabilizing the assembly of subunits (13). There are several isoforms of at least subunits VIa, VIIa and VIII which are tissueand species-specific, resulting in different isozymes. A liver (l) and a heart (h) isoform have been found in VIa, VIIa and VIII subunits, and the h-isoform is also present in skeletal muscles (13). Rat brown fat has both isoforms (13).
The fact that ovariectomy-derived COX activity enhancement was globally reversed by 10 days but not by 2 days of hormonal treatments suggests a relatively slow mechanism through which female sex steroids control COX activity, i.e. oestradiol and progesterone most probably regulate COX by controlling either the actual amount of COX enzyme or its turnover. Certainly, this type of regulation has been demonstrated in COX from different organisms, although a detailed study about factors controlling mammalian COX is still lacking $(13,14)$. Moreover, the presence of different isoforms in heart and muscle (h-isoform) and in liver 
STOMACH

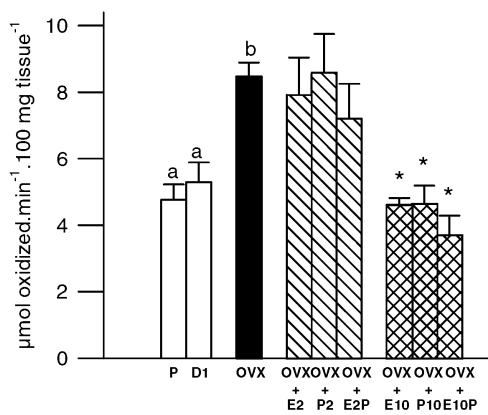

RECTUM

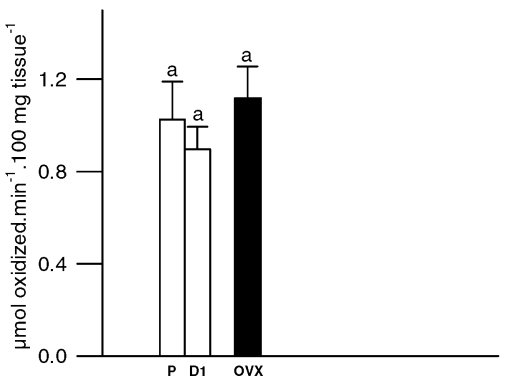

DUODENUM

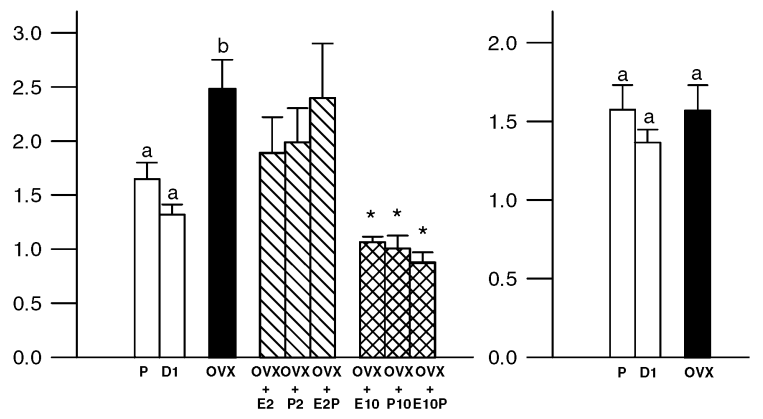

UTERUS

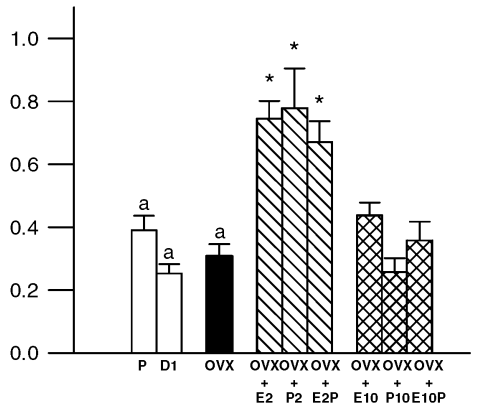

Figure 3 COX activity in smooth muscles from female cold-acclimated rats: intact in proestrus (P) and diestrus I (DI); ovariectomized (OVX); ovariectomized and treated for 2 days or for 10 days with oestradiol (OVX $+\mathrm{E} 2$ and OVX $+\mathrm{E} 10$ respectively), progesterone $(\mathrm{OVX}+\mathrm{P} 2$ and $\mathrm{OVX}+\mathrm{P} 10$ respectively) or oestradiol and progesterone (OVX $+\mathrm{E} 2 \mathrm{P}$ and $\mathrm{OVX}+\mathrm{E} 10 \mathrm{P}$ respectively). In intact rats, COX activity values were unchanged during the oestrous cycle. Ovariectomy induced an increase in stomach and duodenum, an increase that was reversed by 10 days of hormonal treatments. In uterus, 2 days of hormonal treatments induced the opposite effect, i.e. an increase in COX activity. Bars are means \pm S.E. of six or seven animals per group. Among groups $P, D I$ and OVX, bars with different superscript letters are statistically different $(P<0.05$, one-way ANOVA); among OVX groups, *indicates a statistical difference with respect to the OVX group $(P<0.05$, two-way ANOVA).

(l-isoform) could explain why ovariectomy does not affect liver COX activity while increasing the one in most muscles. Therefore, the ovariectomy results suggest that female sex steroids impose a long-term regulation upon oxidative capacity on thermogenic tissues by regulating the synthesis or turnover of regulatory subunits. This does not exclude a control upon the catalytic units in other models, since it has been shown that oestradiol increases mRNA of subunit II in pituitary tumour cell cultures (15).

Although ovariectomy results suggest a long-term control, the oestrous cycle experiment suggests a superimposed short-term control mediated allosterically. Certainly, during the oestrous cycle, the variation of sex steroids plasma levels is relatively short-lasting, since each phase has a length of $24 \mathrm{~h}$ or less (9). It is, therefore, unlikely that these hormones are modifying the amount of cellular COX since the enzyme half-life is longer than $24 \mathrm{~h} \mathrm{(16).} \mathrm{A} \mathrm{most} \mathrm{plausible} \mathrm{way} \mathrm{of} \mathrm{control}$ is an allosterically driven regulation of the activity of the enzyme, as already suggested (8). The allosteric control of COX activity is widely documented and can be carried out through different subunits (13). The different influence of oestrous cycle on BAT and soleus muscle

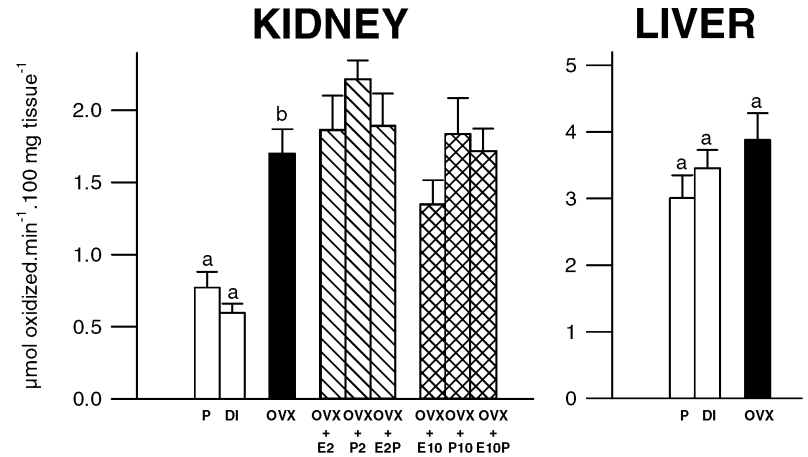

Figure 4 COX activity in kidney and liver from female

cold-acclimated rats: intact in proestrus (P) and diestrus I (DI); ovariectomized (OVX); ovariectomized and treated for 2 days or for 10 days with oestradiol (OVX $+E 2$ and $O V X+E 10$ respectively), progesterone $(\mathrm{OVX}+\mathrm{P} 2$ and $\mathrm{OVX}+\mathrm{P} 10$ respectively) or oestradiol and progesterone $(\mathrm{OVX}+\mathrm{E} 2 \mathrm{P}$ and $\mathrm{OVX}+\mathrm{E} 10 \mathrm{P}$ respectively). In intact rats, COX activity values were unchanged during the oestrous cycle. Ovariectomy induced an increase in COX activity only in kidney, an effect that was still present after 10 days of hormonal treatments. Bars are means \pm S.E. of six or seven animals per group. Among groups $\mathrm{P}, \mathrm{DI}$ and OVX, bars with different superscript letters are statistically different $(P<0.05$, one-way ANOVA). 


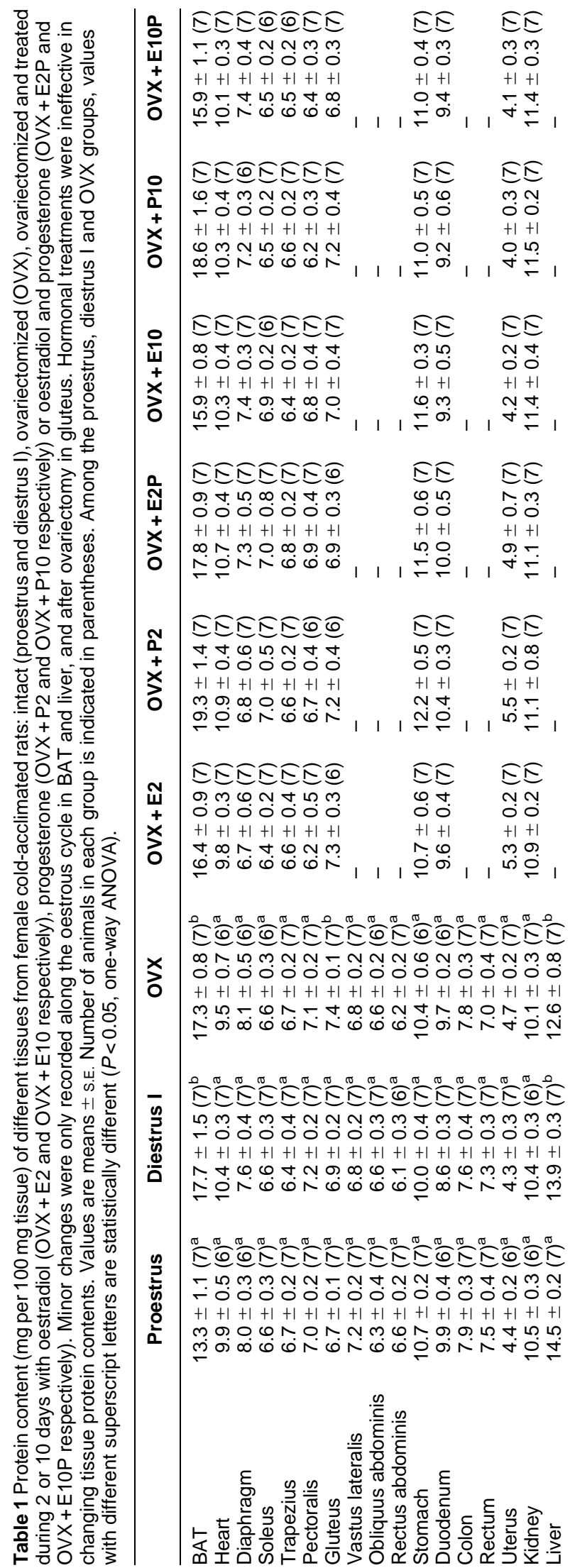

COX activity could, therefore, be explained by the presence of different subunits upon which female sex steroids would exert a differential allosteric influence.

The opposite trends in the short-term influence on BAT and soleus muscle reinforce our previous suggestion that sex steroids exert a coordinated modulation of oxidative capacity in thermogenic tissues (12). In fact, we observed that whole body oxygen consumption, which reflects total oxidative activity, is similar in oestradiol-treated and control animals, in spite of the inhibition induced in BAT oxidative capacity by the oestradiol treatment (6), suggesting that oestradiol would at the same time inhibit BAT and increase thermogenesis in tissues other than BAT. The results of this study agree with such a view, implying a timely organized collaboration of thermogenic tissues, BAT and skeletal muscles, in the full cold-acclimated state. Thus, the lower the BAT oxidative capacity, the greater the muscle one, thus allowing body temperature maintenance. Nonetheless, the fact that the short time influence (as revealed by oestrus cycle experiments) was found exclusively on the soleus muscle, together with the small mass of this muscle (about $400 \mathrm{mg}$ with a specific activity of $1.5 \mu \mathrm{mol}$ oxidized/100 mg tissue) compared with the one in BAT (about $1000 \mathrm{mg}$ with a specific activity of $10 \mu \mathrm{mol}$ oxidized/100 mg tissue) argues against our proposal. However, it should be considered that the soleus muscle consists almost entirely of type I or slow-twitch oxidative fibre muscle (17), these fibres relying mainly on aerobic oxidation. Accordingly, it is logical to suppose that the effect seen in the soleus muscle is an effect in type I fibres. However, these fibres are present in a variable proportion in most of the other skeletal muscles (17), although in much lesser proportion than in the soleus muscle. This extends the effect seen in soleus muscle to a much greater amount of muscle fibres. Thus, although the stimulation shown in proestrus in the small mass of soleus muscle could not by itself counteract the inhibition seen in BAT, there is still the possibility that the effect upon the whole pool of type I muscular fibres could be important at the whole organism level.

The fact that both progesterone and oestradiol reversed the ovariectomy-derived enhancement of COX activity indicates that they probably share a common mechanism for controlling COX synthesis or turnover. However, our data do not allow us to speculate about this as it is becoming increasingly clear that steroid hormones do not only act as transcription factors (18) but also, either alone or in combination with their serum binding proteins, through plasma membrane receptors, displaying either direct effects or interacting with other signalling pathways (18-22). Nonetheless, it should be mentioned that brown adipocytes isolated from either oestradiol-treated (23) or progesterone-treated (24) rats showed a lower oxygen consumption than those isolated from control animals, thus reinforcing a similarity in the effects of both hormones. 
In conclusion, ovariectomy increases COX activity in thermogenic tissues, BAT and smooth and skeletal muscles. This increase is reversed with either oestradiol or progesterone which reveals a basal control of female sex steroids in oxidative metabolism. Nonetheless, a superimposed short-term control is also evident from the cyclic changes throughout the oestrous cycle. Since this short-term influence shows opposite directions in BAT than in muscle, it is inferred that both tissues cooperate in body temperature maintenance in cold-acclimated rats.

\section{Acknowledgements}

This work was supported by grant PB93-0430 from DGICYT (Ministerio de Educación y Ciencia, Spain) and 08.6/0007/1998 from Consejería de Educación y Cultura (Comunidad de Madrid, Spain) to M P.

\section{References}

1 Klaus S, Casteilla L, Bouillaud F \& Ricquier D. The uncoupling protein UCP - a membranous mitochondrial ion carrier exclusively expressed in brown adipose tissue. International Journal of Biochemistry 199123 791-801.

2 Block BA. Thermogenesis in muscle. Annual Review of Physiology $199456535-577$.

3 Colquhoun EQ \& Clark MG. Open question: has thermogenesis in muscle been overlooked and misinterpreted? News in Physiological Sciences 19916 256-259.

4 Boss O, Samec S, Paoloni-Giacobino A, Rossier C, Dulloo A Seydoux J et al. Uncoupling protein-3: a new member of the mitochondrial carrier family with tissue-specific expression. FEBS Letters 1997408 39-42.

5 Boss O, Samec S, Dulloo A, Seydoux J, Muzzin P \& Giacobino J-P. Tissue-dependent upregulation of rat uncoupling protein-2 expression in response to fasting or cold. FEBS Letters 1997 412 111-114.

6 Puerta ML, Nava MP, Abelenda M \& Fernández A. Inactivation of brown adipose tissue thermogenesis by oestradiol treatment in cold-acclimated rats. Pflügers Archiv European Journal of Physiology 1990416 659-662.

7 Nava MP, Abelenda M \& Puerta ML. Cold-induced and dietinduced thermogenesis in progesterone-treated rats. Pflügers Archiv European Journal of Physiology 1990415 747-750.

8 Puerta M, Rocha M, González-Covaleda S, McBennet SN \& Andrews JF. Changes in cytochrome oxidase activity in brown adipose tissue during oestrus cycle in the rat. European Journal of Endocrinology $1998139433-437$.

9 Butcher RL, Collins WE \& Fugo NW. Plasma concentration of $\mathrm{LH}, \mathrm{FSH}$, prolactin, progesterone and estradiol- $17 \beta$ throughout the 4-days estrous cycle of the rat. Endocrinology $1974941704-$ 1708.

10 Yonetani T \& Ray GS. Studies on cytochrome oxidase. VI. Kinetics of the aerobic oxidation of ferrocytochrome $\mathrm{c}$ by cytochrome oxidase. Journal of Biological Chemistry $19652403392-3398$.

11 Lowry OH, Rosebrough NJ, Farr AL \& Randall RJ. Protein measurement with the folin phenol reagent. Journal of Biological Chemistry 1951193 265-275.

12 Fernández A, Abelenda M, Nava MP \& Puerta M. Oxygen consumption of oestradiol-treated rats. Pflügers Archiv European Journal of Physiology 1994426 351-353.

13 Kadenbach B \& Reimann A. Cytochrome c oxidase: tissuespecific expression of isoforms and regulation of activity. In Molecular Mechanisms in Bioenergetics, pp 241-263. Ed L Ernster. Amsterdam: Elsevier, 1992.

14 Poyton RO \& McEwen JE. Crosstalk between nuclear and mitochondrial genomes. Annual Review of Biochemistry 199665 563607.

15 Van Itallie CM \& Dannies PS. Estrogen induces accumulation of the mitochondrial ribonucleic acid for subunit II of cytochrome oxidase in pituitary tumor cells. Molecular Endocrinology $19882332-337$.

16 Saikumar P \& Kurup CK. Effect of administration of 2-methyl-4dimethylaminoazobenzene on the half-lives of rat liver mitochondria and cytochrome oxidase. Biochimica et Biophysica Acta 1985 840 127-133.

17 Armstrong RB \& Phelps RO. Muscle fiber type composition of the rat hindlimb. American Journal of Anatomy 1984171 259-272.

18 Weigel NL. Steroid hormone receptors and their regulation by phosphorylation. Biochemical Journal $1996319657-667$.

19 Katzenellenbogen BS, Montano MM, Kraus WL, Aronica SM, Fujimoto $\mathrm{N} \&$ LeGoff P. Estrogen-receptor and antiestrogenreceptor complexes: cell- and promoter-specific effects and interactions with second messenger signalling pathways. In Organ-selective Actions of Steroid Hormones, pp 29-50. Eds DT Baird, G Schütz \& R Krattenmacher. Berlin: Springer-Verlag, 1995.

20 Fortunati N, Fissore F, Comba A, Becchis M, Catalano MG, Fazzari A et al. Sex steroid-binding protein and its membrane receptor in estrogen-dependent breast cancer: biological and pathophysiological impact. Hormone Research 199645 202-206.

21 Ramirez VD \& Zheng J. Membrane sex-steroid receptors in the brain. Frontiers in Neuroendocrinology $199617402-439$.

22 Soontjens CD, Rafter JJ \& Gustafsson J-A. Ligands for orphan receptors? Journal of Neuroendocrinology 1996150 S241-S257.

23 Puerta M, Abelenda M, Nava MP \& Fernández A. Reduced noradrenaline responsiveness of brown adipocytes isolated from estradiol-treated rats. Canadian Journal of Physiology and Pharmacology $199371858-861$.

24 Abelenda M, Castro C, Venero C \& Puerta M. Reduced oxygen consumption of brown adipocytes isolated from progesteronetreated rats. Canadian Journal of Physiology and Pharmacology $1994721226-1230$

Received 26 April 1999

Accepted 4 August 1999 